This document is confidential and is proprietary to the American Chemical Society and its authors. Do not copy or disclose without written permission. If you have received this item in error, notify the sender and delete all copies.

\title{
Bottom-Up Synthesis of Liquid-Phase-Processable Graphene Nanoribbons with Near-Infrared Absorption
}

\begin{tabular}{|r|l|}
\hline Journal: & ACS Nano \\
\hline Manuscript ID: & nn-2014-049014.R2 \\
\hline Manuscript Type: & Article \\
\hline Date Submitted by the Author: & 21 -Oct-2014 \\
\hline Lomplete List Authors: & $\begin{array}{l}\text { Narita, Akimitsu; Max-Planck-Institute for Polymer Research, } \\
\text { Verzhbitskiy, Ivan; Free University Berlin, Department of Physics } \\
\text { Frederickx, Wout; Katholieke Universiteit Leuven, Department of Chemistry } \\
\text { Mali, Kunal; Katholieke Universiteit Leuven, Department of Chemistry } \\
\text { Jensen, Soeren; Max Planck Institute for Polymer Research, Molecular } \\
\text { Spectroscopy } \\
\text { Hansen, Michael Ryan; Max-Planck-Institute for Polymer Research, } \\
\text { Bonn, Mischa; Max Planck Institute for Polymer Research, Molecular } \\
\text { spectroscopy } \\
\text { De Feyter, Steven; KU Leuven, Department of Chemistry } \\
\text { Casiraghi, Cinzia; University of Manchester, School of Chemistry } \\
\text { Feng, Xinliang; Dresden University of Technology, Molecular Functional } \\
\text { Materials } \\
\text { Müllen, Klaus; Max-Planck-Institute for Polymer Research, }\end{array}$ \\
\hline
\end{tabular}




\section{Bottom-Up Synthesis of Liquid-Phase-Processable}

\section{Graphene Nanoribbons with Near-Infrared}

\section{Absorption}

Akimitsu Narita, ${ }^{\dagger}$ Ivan A. Verzhbitskiy, ${ }^{\star}$ Wout Frederickx, ${ }^{\S}$ Kunal S. Mali, ${ }^{\S}$ Soeren Alkaersig Jensen, ${ }^{\dagger}, \|$ Michael Ryan Hansen, ${ }^{\dagger} \perp$ Mischa Bonn, ${ }^{\dagger}$ Steven De Feyter, ${ }^{\S}$ Cinzia Casiraghi, ${ }^{+, \|}$ Xinliang Feng, ${ }^{* \dagger}$ and Klaus Müllen ${ }^{* \dagger}$

${ }^{\dagger}$ Max Planck Institute for Polymer Research, Ackermannweg 10, D-55128 Mainz, Germany

Department of Physics, Free University Berlin, Arnimalle 14, 14195 Berlin, Germany

${ }^{\S}$ Division of Molecular Imaging and Photonics, Department of Chemistry, KU LeuvenUniversity of Leuven, Celestijnenlaan, 200 F, B-3001 Leuven, Belgium

"FOM Institute AMOLF, Science Park 104, 1098 XG Amsterdam, The Netherlands

${ }^{\perp}$ Interdisciplinary Nanoscience Center (iNANO) and Department of Chemistry, Aarhus University, Gustav Wieds Vej 14, DK-8000 Aarhus C, Denmark

"School of Chemistry, Manchester University, Oxford Road, Manchester, M139PL, United Kingdom

*Address conrrespondence to muellen@mpip-mainz.mpg.de; feng@mpip-mainz.mpg.de. 


\begin{abstract}
KEYWORDS: graphene nanoribbon · cyclodehydrogenation $\cdot$ Diels-Alder reaction · bandgap engineering $\cdot$ near-infrared absorption
\end{abstract}

\begin{abstract}
Structurally defined, long $(>100 \mathrm{~nm})$, and low-bandgap $(\sim 1.2 \mathrm{eV})$ graphene nanoribbons (GNRs) were synthesized through a bottom-up approach, enabling GNRs with a broad absorption spanning into the near-infrared (NIR) region. The chemical identity of GNRs was validated by IR, Raman, solid-state NMR, and UV-vis-NIR absorption spectroscopy. Atomic force microscopy revealed well-ordered self-assembled monolayers of uniform GNRs on a graphite surface upon deposition from the liquid phase. The broad absorption of the lowbandgap GNRs enables their detailed characterization by Raman and time-resolved terahertz photoconductivity spectroscopy with excitation at multiple wavelengths, including the NIR region, which provides further insights into the fundamental physical properties of such graphene nanostructures.
\end{abstract}

Graphene nanoribbons (GNRs), nano-strips of graphene, are emerging materials of great promise, which possess non-zero bandgaps in stark contrast to semi-metallic graphene. ${ }^{1,2}$ The electronic properties such as the bandgaps of the GNRs critically depend on their width and edge structures, ${ }^{3-6}$ which makes it crucial to precisely control the GNR structures for fundamental studies as well as for future nanoelectronic applications. GNRs are typically prepared through “top-down" approaches such as lithographic patterning of graphene sheets ${ }^{7-9}$ and unzipping of carbon nanotubes, ${ }^{10-12}$ revealing promising electronic properties of the GNRs. However, these methods cannot avoid high structural disorder, especially at the edges of the resulting GNRs, and 
often lack uniformity in the width. On the other hand, "bottom-up" chemical synthesis can provide a variety of structurally well-defined GNRs based on solution-mediated ${ }^{13-19}$ or surfaceassisted $^{20-23}$ cyclodehydrogenation of tailor-made polyphenylene precursors.

Whereas the GNRs previously prepared through the bottom-up methods were limited by the short length $(<50 \mathrm{~nm})^{13-15}$ and/or the necessity of metal surfaces, ${ }^{20-22}$ we have very recently reported a solution synthesis of structurally well-defined GNR 1 with high longitudinal extension over $200 \mathrm{~nm}$ (Figure 1). ${ }^{16}$ We have employed an efficient $A B$-type Diels-Alder polymerization for the preparation of corresponding polyphenylene precursors with exceptionally large molecular weights, followed by intramolecular oxidative cyclodehydrogenation. Notably, GNR 1 shows a defined bandgap, in stark contrast to carbon nanotubes (CNTs) that are unavoidably obtained as a mixture of different diameters and chiralities with a corresponding wide distribution of bandgaps. ${ }^{24,25}$ Nevertheless, the width of GNR $\mathbf{1}$ is limited to $\sim 1 \mathrm{~nm}$, corresponding to a bandgap of $\sim 1.9 \mathrm{eV}$, with an optical absorption only up to $\sim 650 \mathrm{~nm}$. $^{16}$

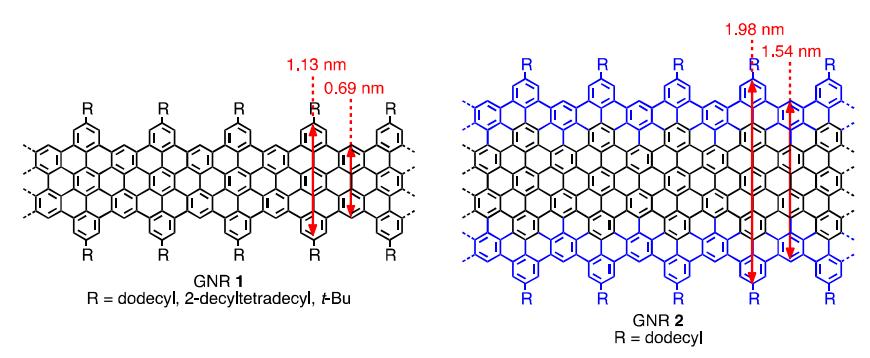

Figure 1. Structures of GNRs 1 and 2. Geometric dimensions were derived from Merck Molecular Force Field 94 (MMFF94) calculations.

For the future development of GNR-based electronics and opto-electronics, it is highly important to tune the GNR width and thus the bandgaps, while maintaining the large longitudinal extension. ${ }^{5,} 26$ Specifically, a smaller bandgap leads to absorption over a wider range of 
wavelengths, possibly including the near-infrared (NIR) region, which opens up possibilities for various applications, including photovoltaics. ${ }^{5,} 27$ Moreover, GNRs with NIR absorption are desirable to spectroscopically obtain an in-depth understanding on the basic physical properties of the GNRs. For instance, Raman measurements at multiple wavelengths provide information on the phononic and electronic dispersions of the GNRs, based on the resonant nature of the Raman scattering process in GNRs. ${ }^{28}$ In this article, we describe the synthesis and characterization of GNR 2 with an extended width of $\sim 2 \mathrm{~nm}$ and lengths exceeding $100 \mathrm{~nm}$ (Figure 1). GNR 2 demonstrates a low and well-defined bandgap of $\sim 1.2 \mathrm{eV}$ with broad optical absorption, extending into the NIR region, in contrast to the relatively large bandgap of GNR $\mathbf{1}$. Such GNRs with NIR absorption allow for in-depth investigations using multi-wavelength Raman spectroscopy and ultrafast terahertz $(\mathrm{THz})$ photoconductivity measurements.

\section{RESULTS AND DISCUSSION}

Bottom-up solution synthesis of GNR 2. In order to synthesize GNR 2 through an efficient $A B$-type Diels-Alder polymerization, a laterally extended monomer 11 was designed, with a cyclopentadienone moiety and an ethynyl group as the conjugated diene and the dienophile, respectively (Scheme 1). 2-Bromo-5-methylbiphenyl (4) was prepared by selective Suzuki coupling of 4-bromo-3-iodotoluene (3) with phenylboronic acid at $60{ }^{\circ} \mathrm{C}$, and then brominated with an excess amount of $\mathrm{N}$-bromosuccinimide (NBS) to give a mixture of 2-bromo-5bromomethylbiphenyl (5) and 2-bromo-5-dibromomethylbiphenyl. The crude mixture was subsequently treated with $\mathrm{HPO}(\mathrm{OEt})_{2}$ and $i$ - $\mathrm{Pr}_{2} \mathrm{NEt}$ for selective debromination at the benzylic $\operatorname{position}^{29}$ to afford 5 in $67 \%$ yield for two steps. Phase-transfer carbonylation of 5 with $\mathrm{Fe}(\mathrm{CO})_{5}$ provided bis(bromobiphenyl)acetone 6 in $39 \%$ yield, which was reacted with dodecylphenyl 
boronic acid (7) via Suzuki coupling to obtain bis(o-terphenyl)acetone 8 in $76 \%$ yield. Finally, Knoevenagel condensation of $\mathbf{8}$ with benzil $\mathbf{9}^{17}$ and subsequent removal of the triisopropylsilyl protecting group afforded the laterally extended monomer 11 in $47 \%$ yield for two steps.

Scheme 1. Synthetic route toward GNR 2.
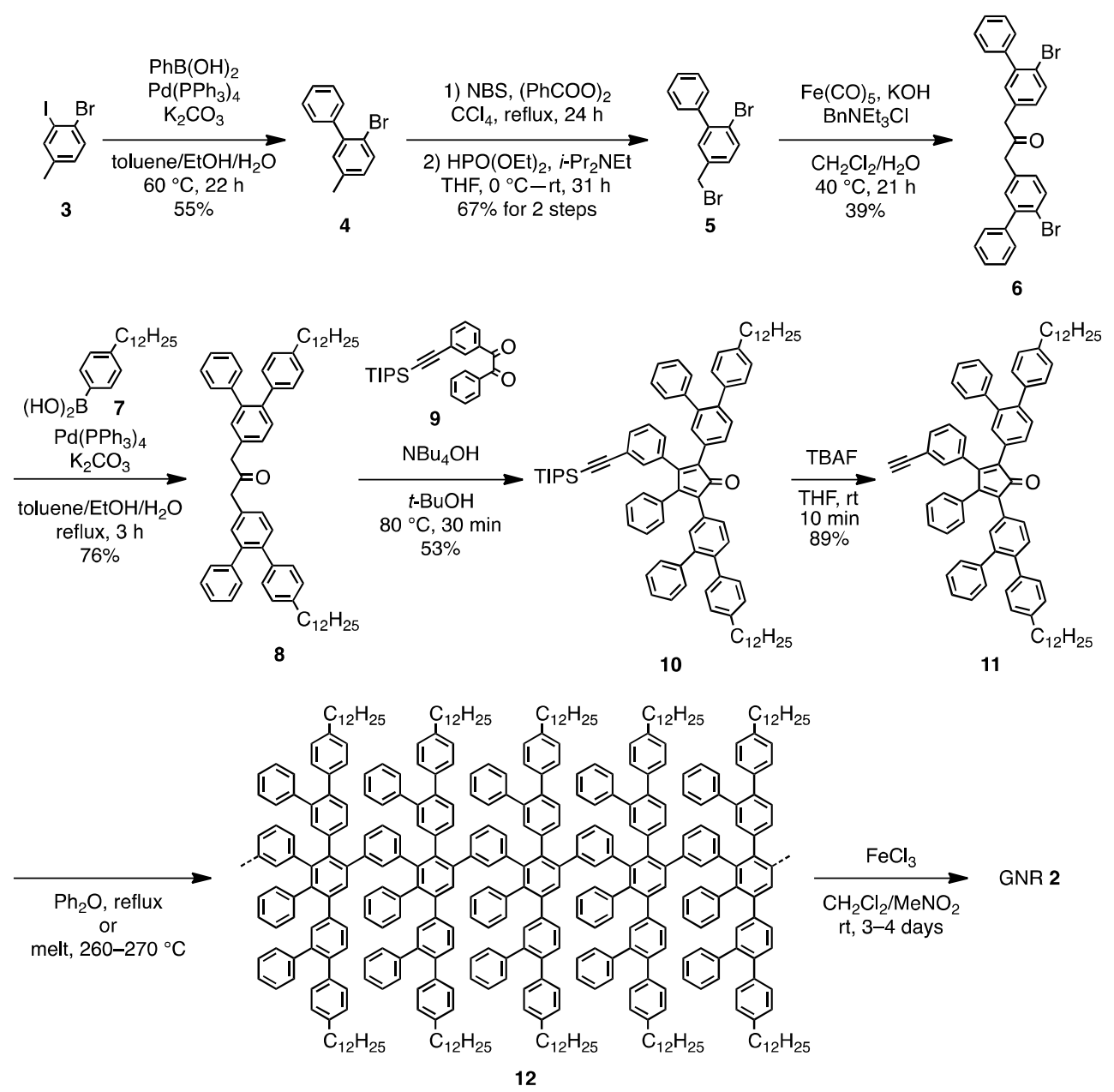

$A B$-type Diels-Alder polymerization of monomer 11 was performed either by refluxing in diphenyl ether or heating at $260-270{ }^{\circ} \mathrm{C}$ in a melt to provide polyphenylene precursor $\mathbf{1 2}$. Precursor 12 contained small oligomers upon preparation, according to size exclusion 
chromatography (SEC) analysis, but such oligomers could be removed by fractionation with recycling preparative SEC (see Supporting Information for details). When monomer 11 was refluxed in diphenyl ether at a concentration of $221 \mathrm{mM}$, precursor 12-I with weight-average molecular weight $\left(M_{\mathrm{w}}\right)$ of $42000-78000 \mathrm{~g} \cdot \mathrm{mol}^{-1}$ and polydispersity index (PDI) of 1.4-1.5 was obtained after the removal of the smaller oligomers, based on SEC analysis against poly(paraphenylene) (PPP) and polystyrene (PS) standards (Table S1, entry 1'). Although the $M_{\mathrm{w}}$ values obtained by SEC analysis are only estimations based on the PPP and PS standard calibrations, they are useful for effectively comparing the molecular weights of different polyphenylene precursors. $^{16}$

Notably, the polymerization of monomer $\mathbf{1 1}$ in a melt led to precursor 12-II with a significantly higher $M_{\mathrm{w}}$ of $230000-550000 \mathrm{~g} \cdot \mathrm{mol}^{-1}$ with PDI of 2.8-3.8 after removing the smaller oligomers by the preparative SEC (Table S1, entry 3'). This result is consistent with previous reports that polymers with larger molecular weight can be obtained when the DielsAlder polymerization is carried out at higher monomer concentrations or in a melt. ${ }^{16,30,31}$ This $M_{\mathrm{w}}$ is comparable to the largest value obtained for the precursor of GNR $\mathbf{1},{ }^{16}$ and demonstrates the extremely high efficiency of $A B$-type Diels-Alder polymerization, also for the laterally extended system.

Subsequently, polyphenylene precursors 12-I and 12-II were "graphitized" into GNRs 2-I and 2-II, respectively, through oxidative cyclodehydrogenation with iron (III) chloride (Scheme 1). Precursor 12 contained numerous regioisomers, considering the two possible molecular orientations of unsymmetrical monomer 11 upon each cycloaddition step (Figure S4). Nevertheless, all the isomers lead to the identical GNR structure upon planarization (Figure S4). Based on the $M_{\mathrm{w}}$ of precursors 12-I and 12-II, the average lengths of GNRs 2-I and 2-II could 
be estimated to be ca. 30-56 and 160-390 nm, respectively. Although these values are only estimations, they indicated that GNR 2-II is at least around three times longer than GNR 2-I.

\section{FTIR and NMR spectroscopic characterization of precursor 12 and GNR 2. The} efficiency of the cyclodehydrogenation was first examined by Fourier transform infrared (FTIR) spectroscopic analysis (Figures 2 and S5). A comparison of the FTIR spectra of precursor 12 and GNR 2 revealed the strong attenuation of the signal triad from aromatic $\mathrm{C}-\mathrm{H}$ stretching vibrations at 3025,3058 , and $3082 \mathrm{~cm}^{-1}$, as well as the disappearance of out-of-plane (opla) C-H deformation bands at 698,773 , and $893 \mathrm{~cm}^{-1}$, which are characteristic for mono- and disubstituted benzene rings (Figure 2). ${ }^{15,16}$ Moreover, the band appearing at $863 \mathrm{~cm}^{-1}$ could be assigned to the opla band from the aromatic C-H moiety at the cove position (see Figure 4d), supporting the successful conversion of precursor 12 into GNR 2. ${ }^{16,32}$
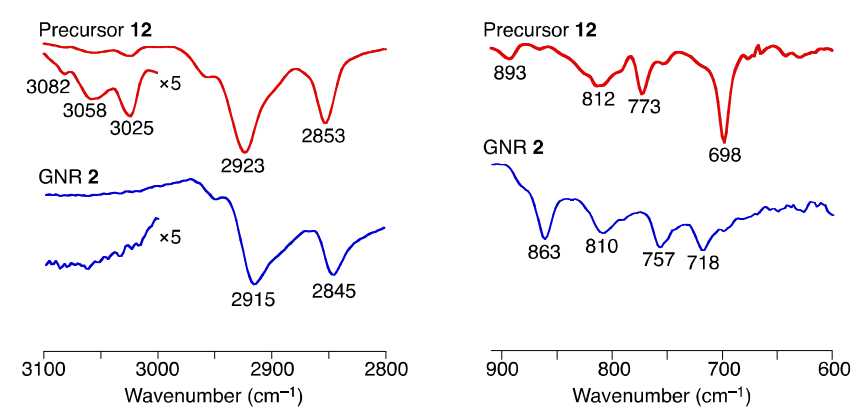

Figure 2. Representative FTIR spectral regions of polyphenylene precursor 12 (red) and GNR 2 (blue).

Solid-state ${ }^{1} \mathrm{H}$ and ${ }^{13} \mathrm{C}$ magic-angle spinning (MAS) NMR spectroscopy ${ }^{33,34}$ was employed to further characterize precursor 12 and GNR 2 (Figures 3 and 4). The overall ${ }^{1} \mathrm{H}$ line width from the aromatic protons of precursor 12 , centered at $\sim 6.8 \mathrm{ppm}$, was similar in solution and in the 
solid state, which indicated its relatively flexible structure (Figure $3 \mathrm{a}$ and $\mathrm{b}$ ). In contrast, the ${ }^{1} \mathrm{H}$ MAS NMR spectrum of GNR 2 showed significantly broadened and shifted aromatic and aliphatic ${ }^{1} \mathrm{H}$ resonances, corroborating the rigid and planar feature of GNR 2 with extended $\pi$ conjugation (Figure 3c). ${ }^{35-37}$

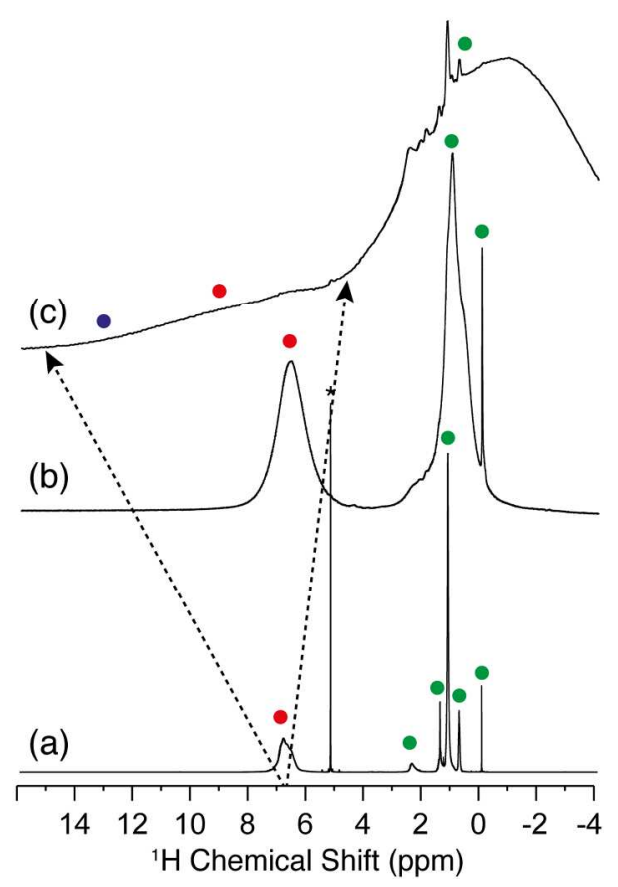

Figure 3 Liquid- and solid-state ${ }^{1} \mathrm{H}$ NMR spectra of (a) precursor 12 dissolved in $\mathrm{CD}_{2} \mathrm{Cl}_{2}$, and (b), (c) solid samples of precursor 12 and GNR 2, respectively. The liquid-state NMR spectrum in (a) was acquired at $7.05 \mathrm{~T}\left(300.13 \mathrm{MHz}\right.$ for $\left.{ }^{1} \mathrm{H}\right)$, while the solid-state NMR experiments in (b) and (c) were recorded at 20.0 T (850.26 MHz for $\left.{ }^{1} \mathrm{H}\right)$ using a MAS frequency of $59524 \mathrm{~Hz}$. The asterisks in (a) mark the residual proton signal of $\mathrm{CD}_{2} \mathrm{Cl}_{2}$. The dashed arrows illustrate the large spread of ${ }^{1} \mathrm{H}$ chemical shifts in GNR $\mathbf{2}$ compared to $\mathbf{1 2}$ in solution.

2D ${ }^{1} \mathrm{H}-{ }^{1} \mathrm{H}$ double quantum-single quantum (DQ-SQ) MAS NMR correlation experiments were also performed to probe the spatial proximity between different protons via dipole-dipole 
interactions (Figure $4 \mathrm{a}$ and b). ${ }^{38,39}$ In agreement with the relatively flexible structure, the $2 \mathrm{D}{ }^{1} \mathrm{H}$ ${ }^{1} \mathrm{H}$ DQ-SQ MAS NMR spectrum of precursor 12 only showed narrow ${ }^{1} \mathrm{H}-{ }^{1} \mathrm{H}$ correlation signals between the aromatic protons as well as between the aromatic and aliphatic protons (Figure 4a). On the other hand, GNR 2 displayed a broad, stretched, and split ridge of ${ }^{1} \mathrm{H}-{ }^{1} \mathrm{H}$ correlation signals close to the spectrum diagonal ( $\mathrm{SQ}_{\text {iso }} \sim 7-15 \mathrm{ppm}$ ), in addition to a broad range of ${ }^{1} \mathrm{H}-{ }^{1} \mathrm{H}$ correlation signals between aromatic and aliphatic protons ( $\left.\mathrm{SQ}_{\text {iso }} \sim 4-12 \mathrm{ppm}\right)$ (Figure $\left.4 \mathrm{~b}\right)$. On the basis of the correlations to the aliphatic protons, the lower- and higher-frequency parts of the aromatic signals could be assigned to the outer (red circle) and the inner (blue circle) proton, respectively (Figure $4 \mathrm{~b}$ and $\mathrm{d}$ ). Interestingly, the spectral features observed for GNR $\mathbf{2}$ are broader than those of narrower GNR $1,{ }^{16}$ most likely due to larger aromatic currents induced by the extended aromatic cores.

Additionally, a 1D ${ }^{13} \mathrm{C}\left\{{ }^{1} \mathrm{H}\right\}$ REcoupled Polarization-Transfer Heteronuclear Single Quantum Correlation (REPT-HSQC) spectrum of GNR 2 was measured (Figure 4c). ${ }^{40}$ By using a short recoupling time, only ${ }^{13} \mathrm{C}$ signals from the carbons situated at and near the edges were observed. The spectrum showed three relatively broad ${ }^{13} \mathrm{C}$ signals, which could be assigned to aliphatic side chains $(\sim 30 \mathrm{ppm})$ as well as aromatic $\mathrm{CH}(\sim 124 \mathrm{ppm})$ and quaternary aromatic carbons ( $\sim 139 \mathrm{ppm})$ at or close to the edges, based on their chemical shifts and intensities. 

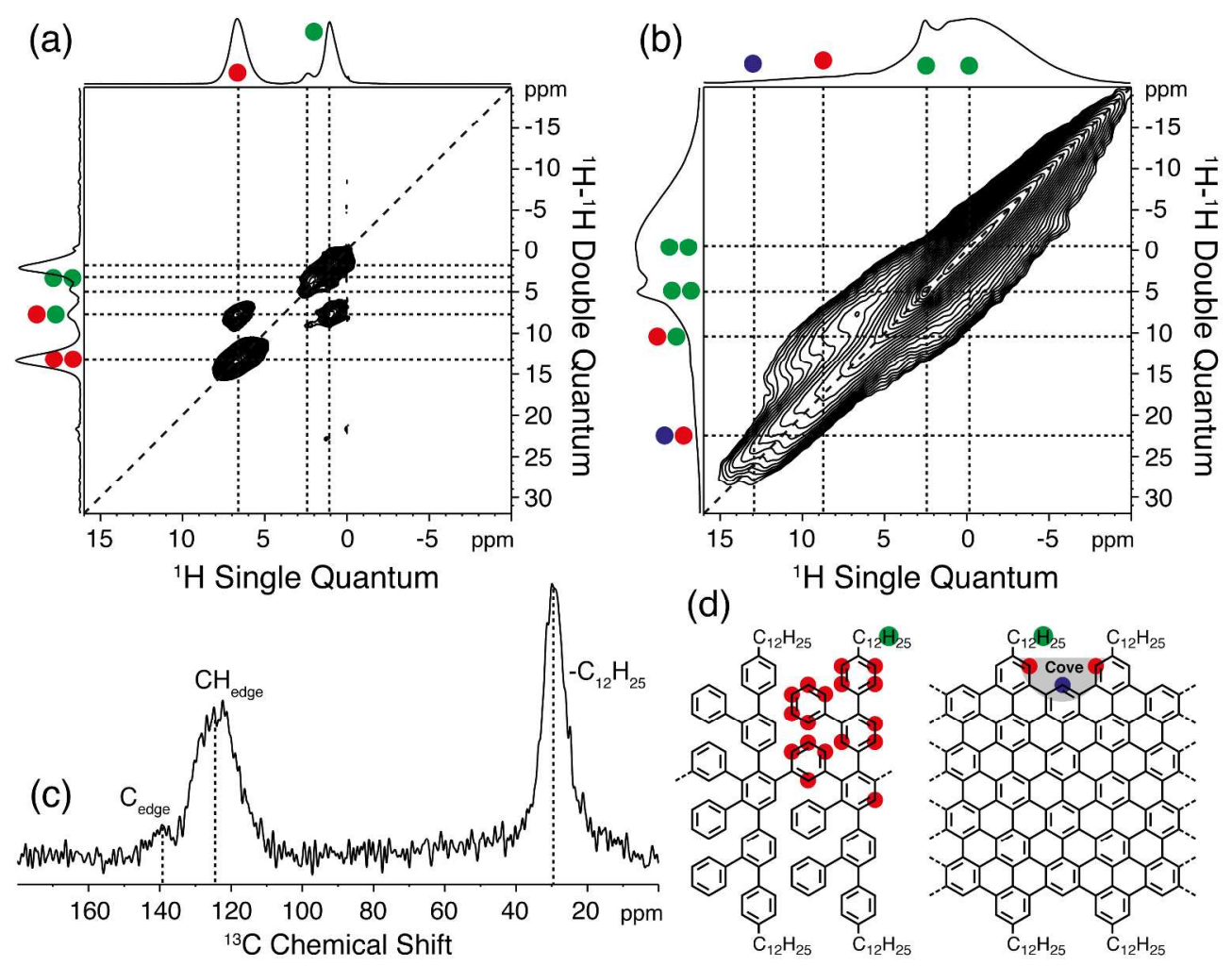

Figure 4 Solid-state NMR characterization of precursor 12 and GNR 2. The $2 \mathrm{D}{ }^{1} \mathrm{H}-{ }^{1} \mathrm{H}$ DQ-SQ correlation spectra of (a) precursor 12 and (b) GNR 2 were recorded using a MAS frequency of $59524 \mathrm{~Hz}$ and two rotor periods of DQ recoupling. (c) $1 \mathrm{D}{ }^{13} \mathrm{C}\left\{{ }^{1} \mathrm{H}\right\}$ REPT-HSQC spectrum recorded with a short REDOR recoupling period ${ }^{40}$ of two rotor periods. (d) Assignment scheme.

UV-vis-NIR absorption and Raman spectroscopic analysis of GNR 2. Thanks to the long alkyl chains densely installed on the peripheral positions, GNR 2 could be dispersed in organic solvents such as tetrahydrofuran (THF), chlorobenzene, and ortho-dichlorobenzene (ODCB) under mild sonication. Some of the GNR powders remained undispersed even after extended sonication, which was similar to the observation during the debundling of $\mathrm{CNTs}^{41}$ as well as the exfoliation of graphene. ${ }^{42}$ This was presumably due to decreasing dispersibility of longer GNRs and/or stronger packing in some of the GNR aggregates, which could not be overcome by the 
mild sonication. Nevertheless, larger aggregates could be removed by sedimentation or filtration through a syringe filter (pore size: $5 \mu \mathrm{m}$ ), leaving pale blue dispersions with typical concentrations of approximately $0.06 \mathrm{mg} / \mathrm{mL}$ without visible particles (Figure 5a, inset). The relatively higher concentration of GNR 2 than the reported value for GNR 1, i.e. $\sim 0.01 \mathrm{mg} / \mathrm{mL},{ }^{16}$ is most probably due to inclusion of larger aggregates in dispersions of GNR 2. GNR 2 showed apparently lower dispersibility compared to that of GNR 1 due to stronger $\pi-\pi$ interaction between the extended aromatic cores. Nevertheless, it was still possible to spectroscopically characterize GNR 2 in a dispersion as well as to process it from a liquid phase for microscopic analyses (see below).

UV-vis-NIR absorption spectroscopic analysis of GNR 2 was thus performed on a dispersion in THF (Figure 5a). GNRs 2-I and 2-II showed comparable spectra, indicating that the longitudinal extension of both 2-I and 2-II was sufficient to reach the bandgap of the infinite GNR (Figure S6a). ${ }^{16}$ Compared to the absorption spectrum of GNR 1, that of GNR 2 displayed a significantly broader absorption extending into the NIR region, with an absorption maximum at $660 \mathrm{~nm}$. Whereas the absorption spectrum in 1,2,4-trichlorobenzene (TCB) was identical to the one in THF, the spectrum measured in ODCB displayed a shifted absorption maximum at 645 $\mathrm{nm}$ (Figure S6b). This observation suggests a different degree of GNR aggregation in these solvents, affecting the absorption profile. ${ }^{26}$ The absorption edge was determined as the Xintercept by extrapolation to be $1000 \pm 20 \mathrm{~nm}$, considering the variation in the absorption spectra in TCB, THF, and ODCB. Although the effect of the aggregation cannot completely be excluded, the optical bandgap of GNR 2 could be estimated from this absorption edge to be $1.24 \pm 0.03 \mathrm{eV}$, which was in good agreement with the theoretical bandgap of $1.18 \mathrm{eV}$ obtained using density functional theory (DFT) calculations. ${ }^{5}$ This optical bandgap is considerably smaller 
than the $\sim 1.88 \mathrm{eV}^{16}$ reported for GNR 1 , clearly demonstrating the lowering of the bandgap upon increasing the lateral extension.
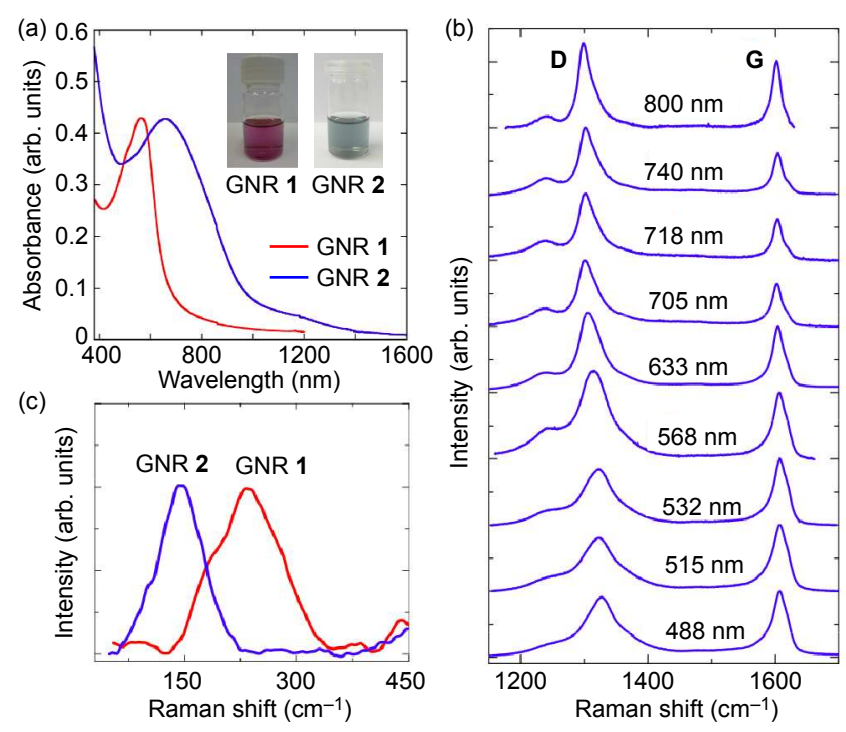

Figure 5 (a) Normalized UV-vis-NIR absorption spectra of GNRs 1 and $\mathbf{2}$ in THF, inset: photographs of dispersions of GNRs 1 and $\mathbf{2}$ in THF. (b) Raman spectra of GNR 2 measured from $488 \mathrm{~nm}(2.54 \mathrm{eV})$ to $800 \mathrm{~nm}(1.55 \mathrm{eV})$ on a powder sample with laser power below 0.1 mW. (c) Acoustic region of the Raman spectra of GNRs 1 and 2, measured at $515 \mathrm{~nm}(2.41 \mathrm{eV})$ and $633 \mathrm{~nm}(1.96 \mathrm{eV})$, respectively, showing the RBLM peaks.

The Raman spectrum of GNR 2 displayed first-order D and G peaks (Figure 5b), which was qualitatively similar to the Raman spectra of previously reported bottom-up synthesized GNRs. ${ }^{15}$, 16, 18-20 GNR 2 with a width of $\sim 2 \mathrm{~nm}$ demonstrated a distinct peak in the low-frequency region (at $\sim 170 \mathrm{~cm}^{-1}$ ) that was assigned to the so-called radial breathing-like mode (RBLM) (Figure 5c). ${ }^{16,20}$ The observation of this peak at a lower position, compared to that of the narrower GNR 1 with a width of $\sim 1 \mathrm{~nm}$ (at $\sim 235 \mathrm{~cm}^{-1}$ ), ${ }^{16}$ further verified the uniform and laterally extended 
structure of GNR 2, since the RBLM peak is expected to shift to lower wavenumbers for increasing width, in analogy to the radial breathing mode of carbon nanotubes. ${ }^{43}$ Note however that the exact RBLM peak position may also be affected by the specific edge geometry and functional groups at the edges.

Profiting from the low bandgap for GNR 2, for the first time, we were able to investigate the Raman spectra of structurally defined GNRs over the visible and NIR energy ranges (Figure 5c), without any interference from the strong photoluminescence signals. This is in stark contrast to GNR 1, which could be measured only up to $633 \mathrm{~nm}^{44}$ The D peak of GNR 2 shifted to lower wavenumber with increasing excitation wavelength. Notably, a small peak could clearly be observed at the left side of the D peak with NIR excitation, whereas it appeared as a shoulder of the D peak at smaller wavelengths. A shift of the RBLM was also observed, depending on the excitation wavelength. Careful interpretations of these observations, involving extensive theoretical studies, are expected to provide deeper insight into the phonon and electronic dispersions of the GNRs, which will be reported elsewhere.

Ultrafast THz photoconductivity analysis of GNR 2. The ultrafast photoconductivity of GNR 2 dispersed in TCB was investigated through non-contact, time-resolved optical pump THz probe spectroscopy. ${ }^{45}$ Whereas a study of the intrinsic electronic properties using device measurements is challenging, $\mathrm{THz}$ spectroscopy provides a straightforward and non-invasive approach to evaluate the photoconductivity within individual GNRs on ultrashort timescales. ${ }^{16,46}$ The lower bandgap of GNR 2 enabled photoconductivity studies over a wider range of pump photon energies as compared to GNR 1. Figure 6 shows the probe frequency resolved- (a) and time dependent ( $b$ and c) complex conductivity of GNR 2 excited with a wavelength of $400 \mathrm{~nm}$ $(3.10 \mathrm{eV})$ and $800 \mathrm{~nm}(1.55 \mathrm{eV})$; both the frequency-dependent conductivity and the 
conductivity dynamics are similar to those previously reported for GNR 1 at $400 \mathrm{~nm}$ excitation. ${ }^{16,46}$ Remarkably, the time-dependent photoconductivity of GNR 2 measured at 400 and $800 \mathrm{~nm}$ excitation are very similar. For both excitation energies, the dynamics show a quick rise upon excitation followed by a fast decay in both the real and imaginary parts (Figure 6b and c).

The lower optical absorption of GNR 2 due to the limited dispersibility made the measurements more sensitive to losses of pump photons from scattering. The values for the conductivity normalized to excitation density in Figure $6 \mathrm{a}$ should therefore be taken as conservative estimates. Nevertheless, the observed magnitude of the real conductivity just after excitation indicated a high photoconductivity of GNR 2. Uncorrected for scattering of the pump light, the inferred photoconductivity both at $400 \mathrm{~nm}$ and $800 \mathrm{~nm}$ excitation is comparable to that of GNR 1, ${ }^{16,46}$ implying that the true photoconductivity of the broader GNR $\mathbf{2}$ is somewhat larger than that of the narrower GNR 1, as expected, ${ }^{3}$ given the smaller bandgap of GNR 2. Further, the frequency resolved photoconductivity of GNR 2 at 400 and $800 \mathrm{~nm}$ excitation yielded a very similar shape, but with a slightly higher value for the scaled conductivity at 400 $\mathrm{nm}$, suggesting a higher excitation quantum yield at $400 \mathrm{~nm}$ than at $800 \mathrm{~nm}$.

Clearly, the THz spectroscopy measurements on GNR 2 demonstrate the generation of conductive charge-carriers with excitation at both $800 \mathrm{~nm}$ and $400 \mathrm{~nm}$, whereas GNR 1 showed a response only at $400 \mathrm{~nm}$. These results show that GNR 2 can be studied by pump-probe spectroscopy techniques at a broader range of excitation wavelengths, allowing more detailed investigations, for example, on the process of carrier multiplication ${ }^{47-49}$ in the GNRs. 
(a)

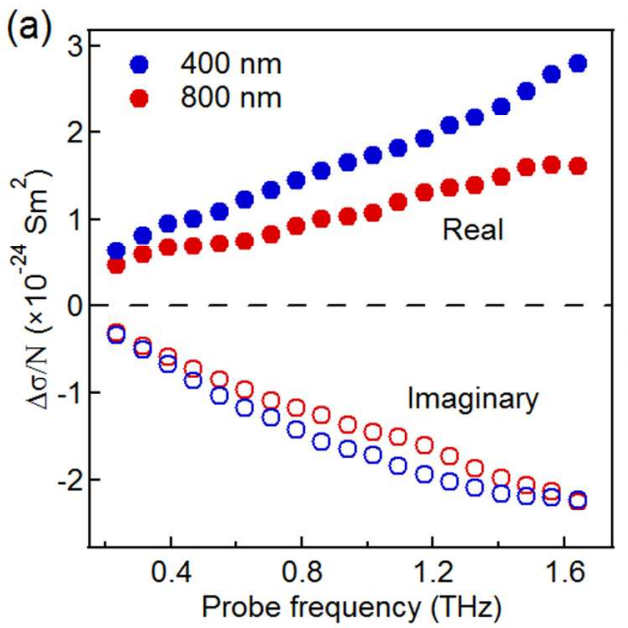

(b)

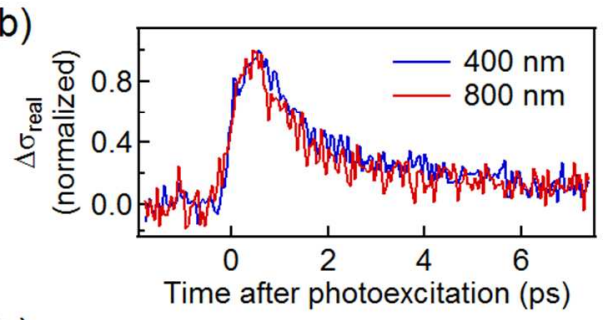

(c)

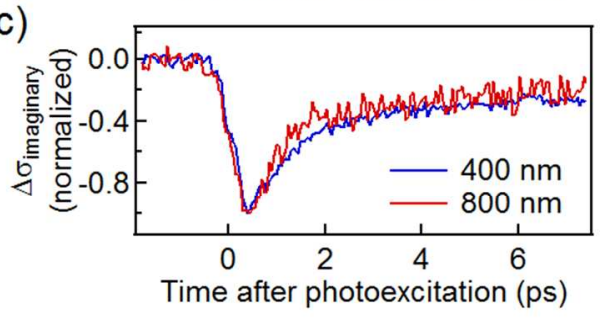

Figure 6 (a) Frequency-resolved, photoinduced complex conductivity of GNR 2 dispersed in TCB, scaled to the initial excitation density $N$, measured $0.5 \mathrm{ps}$ after excitation by a $400 \mathrm{~nm}$ or $800 \mathrm{~nm}$ pulse. (b) Real and (c) imaginary components of the photoconductivity of GNR 2 as function of time after excitation. Absorbed pump fluence was $3.9 \times 10^{18}$ photons $/ \mathrm{m}^{2}$ at $800 \mathrm{~nm}$ and $4.2 \times 10^{18}$ photons $/ \mathrm{m}^{2}$ at $400 \mathrm{~nm}$.

AFM visualization of GNR 2. Atomic force microscopy (AFM) analysis demonstrated the formation of well-ordered self-assembled monolayers on highly oriented pyrolytic graphite (HOPG), upon deposition of GNR 2-I from a dispersion in hot TCB (120-130 ${ }^{\circ} \mathrm{C}$ ) (Figures 7 and S7). The periodicity of the striped features was $5.0 \pm 0.2 \mathrm{~nm}$ (Figure 7c), which was consistent with the $\sim 4.8 \mathrm{~nm}$ expected lateral dimension of GNR 2 including the alkyl chains (Figure 7d). This observation indicated the adsorption of the GNRs next to each other without stacking or interdigitation of the alkyl chains. Whereas GNR 1 with the dodecyl chains showed partial stacking upon the formation of self-assembled monolayers, ${ }^{16}$ the absence of such stacking for GNR 2 could be explained by the enhanced interaction of the extended aromatic cores with the 
HOPG surface. This result further attested the defined structure of GNR 2 with high uniformity, and displayed its liquid-phase processability.

Highly covered areas extending several hundred nanometers in both lateral and longitudinal directions were observed (Figure S8), suggesting end-to-end packing of the GNRs, given that the average length of GNR 2-I is ca. 30-56 nm. Indeed, the length of the GNRs in isolated small domains was typically ca. 20-70 nm, which is in agreement with the estimated length (Figures 7b and S9). Although monolayers of GNR 2-II could not be visualized using AFM, presumably due to its lower processability, this sample should contain long GNRs extending over $100 \mathrm{~nm}$, considering that GNR 2-II was at least around three times longer than GNR 2-I.
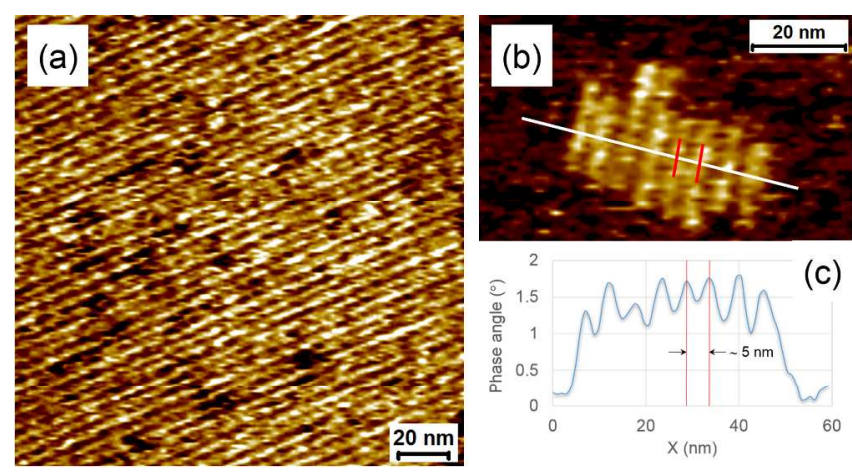

(d)

$\longleftarrow 4.8 \mathrm{~nm} \longrightarrow$

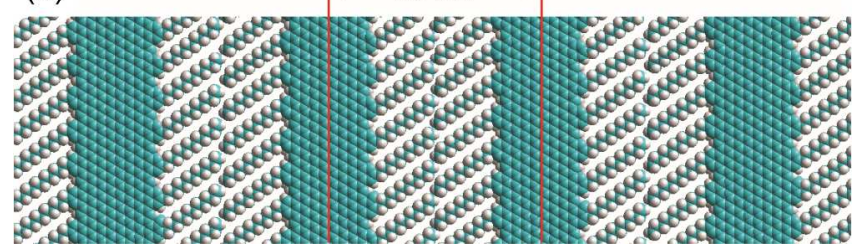

Figure 7 (a), (b) AFM phase images of self-assembled monolayers of GNR 2-I on HOPG (dry film), showing (a) a large domain and (b) a small isolated domain. (c) Representative line profile, which was measured along the white line in the phase image shown in panel b. (d) Molecular model of GNR 2 displaying the packing of the GNRs in the self-assembled monolayer. 


\section{CONCLUSIONS}

A bottom-up synthesis of laterally $(\sim 2 \mathrm{~nm})$ as well as longitudinally $(>100 \mathrm{~nm})$ extended GNRs was achieved based on the $A B$-type Diels-Alder polymerization, displaying a low optical bandgap of approximately $1.24 \mathrm{eV}$. The efficient planarization ("graphitization") of the polyphenylene precursor into the GNRs was corroborated by FTIR, NMR, UV-vis-NIR, and Raman spectroscopy. $\mathrm{THz}$ spectroscopy analysis revealed the high photoconductivity of the laterally extended GNRs. AFM analysis demonstrated the liquid-phase processability of such GNRs albeit with limited dispersibility. Remarkably, the broad absorption of the low-bandgap GNRs enabled their spectroscopic characterizations over the visible to NIR wavelengths, as represented by Raman and pump-probe THz spectroscopy studies. Further detailed spectroscopic investigations, including theoretical studies, are expected to elucidate some of the fundamental physical properties of such graphene nanostructures. Moreover, the synthetic method described potentially allows tuning of the width and bandgap for liquid-phase-processable GNRs by modifying the monomer architecture. This may not only enable the width-dependent studies on the single-GNR transistors but also contribute to the development of GNR-based optoelectronics, such as photodetectors and photovoltaics.

\section{METHODS}

Sample preparation. Full details regarding the synthesis and characterization of all the materials are provided in the Supporting Information.

Solid-state NMR. All solid-state MAS NMR experiments were recorded on a Bruker AVANCE-III 850 spectrometer operating at a Larmor frequency of $850.26 \mathrm{MHz}(20.0 \mathrm{~T})$. The 
experiments employed a $1.3 \mathrm{~mm} \mathrm{H}-\mathrm{X}$ probe (Bruker) using a MAS frequency of $59524 \mathrm{~Hz}$ and a recycle delay of $2 \mathrm{~s}$. The $2 \mathrm{D}{ }^{1} \mathrm{H}-{ }^{1} \mathrm{H}$ DQ-SQ correlation spectra were acquired using the back-toback $(\mathrm{BaBa})$ NMR dipolar recoupling sequence for both excitation and reconversion of DQ coherences followed by a $z$-filter, set to one rotor period, prior to a final $\pi / 2$ pulse for creating transverse observable magnetization. ${ }^{38}$ These experiments used two rotor periods $(33.6 \mu \mathrm{s})$ of $\mathrm{BaBa}$ recoupling blocks to excite/reconvert DQ coherences with 16 scans (full phase cycle) for each of the 256 (precursor 12) and 96 (GNR 2) $\mathrm{t}_{1}$ increments. A $\pi / 2$-pulse length of $1.5 \mu \mathrm{s}$ corresponding to an rf-field strength $\left(v_{\mathrm{rf}}\right)$ of $167 \mathrm{kHz}$ was used. The $1 \mathrm{D}{ }^{13} \mathrm{C}\left\{{ }^{1} \mathrm{H}\right\}$ REPT-HSQC spectrum of GNR 3 was recorded using two rotor periods of Rotational-Echo DOuble Resonance (REDOR) recoupling for 10240 scans. $^{40}$ For the REDOR recoupling, $\pi / 2$-pulses of $1.8 \mu$ s were used for both ${ }^{1} \mathrm{H}$ and ${ }^{13} \mathrm{C}$, while low-power ${ }^{1} \mathrm{H}$ SW-f-TPPM decoupling was applied during acquisition $\left(v_{\mathrm{rf}} \sim 12 \mathrm{kHz}, 0.2 \mathrm{~W}\right) .{ }^{50,51}$ Prior to all experiments the magic angle was checked using $\mathrm{KBr} .{ }^{52}{ }^{1} \mathrm{H}$ and ${ }^{13} \mathrm{C}$ chemical shifts are reported relative to TMS using solid adamantane $\left({ }^{13} \mathrm{C}\right.$, $\left.38.484 \mathrm{ppm} ;{ }^{1} \mathrm{H}, 1.85 \mathrm{ppm}\right)$ as an external reference. ${ }^{53,54}$

Raman spectroscopy. Raman spectroscopy was performed in back-scattering geometry with various spectrometers: a micro-Raman Witec single spectrometer was equipped with $532 \mathrm{~nm}$ SSDP laser; multi-wavelength Raman spectra in the visible range were obtained with a Dilor (Horiba-Jobin Yvon) triple spectrometer, equipped Ar-Kr laser, which possessed several laser lines in the range from $488 \mathrm{~nm}$ to $568 \mathrm{~nm}$, while for red and near-IR range we used a T64000 triple Raman spectrometer (Horiba-Jobin Yvon), equipped with He-Ne laser (633 nm) and the tunable Ti:Saphire laser (Coherent MBR-110) that covered the range from $705 \mathrm{~nm}$ to $915 \mathrm{~nm}$. 
The laser power was kept well below $0.1 \mathrm{~mW}$ to avoid heating or damaging the sample. The laser spot was focused on the grain powder sample with a $100 \times$ long-distance objective.

THz spectroscopy. Sample solutions were placed in a quartz cuvette with an inner thickness of $1 \mathrm{~mm}$. Optical pump-THz probe measurements were performed using the output from a titanium sapphire laser that generated pulses with a central wavelength of $800 \mathrm{~nm}$, and a duration of $\sim 40$ fs with a $1 \mathrm{kHz}$ repetition rate. $400 \mathrm{~nm}$ excitation pulses were generated by frequency doubling the $800 \mathrm{~nm}$ pulses in a Beta Barium Borate (BBO) crystal. Single-cycle probe pulses comprising frequencies in the $0-2 \mathrm{THz}$ range were generated from the $800 \mathrm{~nm}$ pulses in a 0.5 mm thick $<110>$ ZnTe crystal by a process called optical rectification. ${ }^{45}$ Beyond the sample, the transmitted $\mathrm{THz}$ field was detected with a third $800 \mathrm{~nm}$ pulse in a second $<110>$ ZnTe crystal using the electro-optic effect. ${ }^{45}$ By mechanically delaying the detection pulse, the whole waveform of the THz pulse could be measured, and the frequency-resolved conductivity could be obtained as described in Refs. 16 and 46.

AFM analysis. A Multimode AFM with a Nanoscope IV controller (Veeco/Digital Instruments) was employed in intermittent contact mode. Prior to AFM measurements, GNR powder was dispersed in TCB by ultrasonication for $2 \mathrm{~h}$ followed by heating at $120-130{ }^{\circ} \mathrm{C}$ for 15 minutes. A drop of this hot dispersion was applied to a hot HOPG surface, which was also held at $120{ }^{\circ} \mathrm{C}$. After the hot deposition, the HOPG sample was heated for another 15 minutes. The surface was then washed with $2 \mathrm{~mL}$ of TCB and heated again to ensure complete evaporation of the solvent. THF could alternatively be used as a solvent for washing. AFM images were recorded at room temperature and processed using SPIP (Image Metrology) 
software. The width of the GNRs was obtained from analysis of AFM images using two different methods, namely, manual and automated power spectrum density (PSD) analysis. In the first method, the width of the GNRs was measured manually by drawing line profiles across the GNR domains in an AFM image and averaging over many of such domains. In the second method, an automated power spectral density analysis was performed on AFM images, which essentially furnished peaks corresponding to the periodic features present in the AFM images (Figure S10). While the periodicity obtained from manual analysis was $5.0 \pm 0.2 \mathrm{~nm}$, the automated analysis furnished a periodicity of $5.2 \pm 0.2 \mathrm{~nm}$.

Conflict of Interest: Authors declare no competing financial interest.

Acknowledgement. We are grateful to the financial support from European Research Council grant on NANOGRAPH, DFG Priority Program SPP 1459, Graphene Flagship (No. CNECTICT-604391), and European Union Projects UPGRADE, GENIUS, and MoQuaS. C.C. and I.A.V. acknowledge the Humboldt Foundation in the framework of the Sofja Kovalevskaja award. M.R.H. acknowledges the Villum Foundation under the Young Investigator Programme (VKR023122).

Supporting Information Available: Experimental details; additional discussion on the fractionation of polyphenylene precursor 12; SEC profiles; full FTIR spectra; additional UVvis-NIR absorption spectra; additional AFM images; and NMR spectra. This material is available free of charge via the Internet at http://pubs.acs.org. 


\section{REFERENCES AND NOTES}

1. Li, X.; Wang, X.; Zhang, L.; Lee, S.; Dai, H. Chemically Derived, Ultrasmooth Graphene Nanoribbon Semiconductors. Science 2008, 319, 1229-1232.

2. Bai, J.; Huang, Y. Fabrication and Electrical Properties of Graphene Nanoribbons. Mater. Sci. Eng. R-Rep. 2010, 70, 341-353.

3. Obradovic, B.; Kotlyar, R.; Heinz, F.; Matagne, P.; Rakshit, T.; Giles, M. D.; Stettler, M. A.; Nikonov, D. E. Analysis of Graphene Nanoribbons as a Channel Material for Field-Effect Transistors. Appl. Phys. Lett. 2006, 88, 142102.

4. Wang, J.; Zhao, R.; Yang, M.; Liu, Z. Inverse Relationship between Carrier Mobility and Bandgap in Graphene. J. Chem. Phys. 2013, 138, 084701.

5. Osella, S.; Narita, A.; Schwab, M. G.; Hernandez, Y.; Feng, X.; Müllen, K.; Beljonne, D. Graphene Nanoribbons as Low-Band-Gap Donor Materials for Organic Photovoltaics: QuantumChemical Aided Design. ACS Nano 2012, 6, 5539-5548.

6. Ritter, K. A.; Lyding, J. W. The Influence of Edge Structure on the Electronic Properties of Graphene Quantum Dots and Nanoribbons. Nature Mater. 2009, 8, 235-242.

7. Chen, Z.; Lin, Y.; Rooks, M.; Avouris, P. Graphene Nano-Ribbon Electronics. Physica E 2007, 40, 228-232.

8. Han, M.; Özyilmaz, B.; Zhang, Y.; Kim, P. Energy Band-Gap Engineering of Graphene Nanoribbons. Phys. Rev. Lett. 2007, 98, 206805.

9. Abbas, A. N.; Liu, G.; Liu, B.; Zhang, L.; Liu, H.; Ohlberg, D.; Wu, W.; Zhou, C. Patterning, Characterization, and Chemical Sensing Applications of Graphene Nanoribbon Arrays Down to $5 \mathrm{~nm}$ Using Helium Ion Beam Lithography. ACS Nano 2014, 8, 1538-1546.

10. Kosynkin, D. V.; Higginbotham, A. L.; Sinitskii, A.; Lomeda, J. R.; Dimiev, A.; Price, B. K.; Tour, J. M. Longitudinal Unzipping of Carbon Nanotubes to Form Graphene Nanoribbons. Nature 2009, 458, 872-876.

11. Jiao, L.; Zhang, L.; Wang, X.; Diankov, G.; Dai, H. Narrow Graphene Nanoribbons from Carbon Nanotubes. Nature 2009, 458, 877-880.

12. Jiao, L. Y.; Wang, X. R.; Diankov, G.; Wang, H. L.; Dai, H. J. Facile Synthesis of HighQuality Graphene Nanoribbons. Nature Nanotech. 2010, 5, 321-325.

13. Yang, X. Y.; Dou, X.; Rouhanipour, A.; Zhi, L. J.; Rader, H. J.; Müllen, K. TwoDimensional Graphene Nanoribbons. J. Am. Chem. Soc. 2008, 130, 4216-4217.

14. Dössel, L.; Gherghel, L.; Feng, X.; Müllen, K. Graphene Nanoribbons by Chemists: Nanometer-Sized, Soluble, and Defect-Free. Angew. Chem. Int. Ed. 2011, 50, 2540-2543.

15. Schwab, M. G.; Narita, A.; Hernandez, Y.; Balandina, T.; Mali, K. S.; De Feyter, S.; Feng, X.; Müllen, K. Structurally Defined Graphene Nanoribbons with High Lateral Extension. J. Am. Chem. Soc. 2012, 134, 18169-18172.

16. Narita, A.; Feng, X.; Hernandez, Y.; Jensen, S. A.; Bonn, M.; Yang, H.; Verzhbitskiy, I. A.; Casiraghi, C.; Hansen, M. R.; Koch, A. H. R., et al. Structurally Well-Defined and LiquidPhase-Processable Graphene Nanoribbons. Nature Chem. 2014, 6, 126-132.

17. Tan, Y.-Z.; Tang, B.; Parvez, K.; Narita, A.; Osella, S.; Beljonne, D.; Feng, X.; Müllen, K. Atomically Precise Edge Chlorination of Nanographenes and Its Application to Graphene Nanoribbons. Nat. Commun. 2013, 4, 2646.

18. Vo, T. H.; Shekhirev, M.; Kunkel, D. A.; Morton, M. D.; Berglund, E.; Kong, L.; Wilson, P. M.; Dowben, P. A.; Enders, A.; Sinitskii, A. Large-Scale Solution Synthesis of Narrow Graphene Nanoribbons. Nat. Commun. 2014, 5, 3189. 
19. Vo, T. H.; Shekhirev, M.; Kunkel, D. A.; Orange, F.; Guinel, M. J. F.; Enders, A.; Sinitskii, A. Bottom-up Solution Synthesis of Narrow Nitrogen-Doped Graphene Nanoribbons. Chem. Commun. 2014, 50, 4172-4174.

20. Cai, J.; Ruffieux, P.; Jaafar, R.; Bieri, M.; Braun, T.; Blankenburg, S.; Muoth, M.; Seitsonen, A. P.; Saleh, M.; Feng, X., et al. Atomically Precise Bottom-up Fabrication of Graphene Nanoribbons. Nature 2010, 466, 470-473.

21. Bronner, C.; Stremlau, S.; Gille, M.; Brauße, F.; Haase, A.; Hecht, S.; Tegeder, P. Aligning the Band Gap of Graphene Nanoribbons by Monomer Doping. Angew. Chem. Int. Ed. 2013, 52, 4422-4425.

22. Chen, Y.-C.; de Oteyza, D. G.; Pedramrazi, Z.; Chen, C.; Fischer, F. R.; Crommie, M. F. Tuning the Band Gap of Graphene Nanoribbons Synthesized from Molecular Precursors. ACS Nano 2013, 7, 6123-6128.

23. Zhang, Y.; Zhang, Y.; Li, G.; Lu, J.; Lin, X.; Du, S.; Berger, R.; Feng, X.; Müllen, K.; Gao, H.-J. Direct Visualization of Atomically Precise Nitrogen-Doped Graphene Nanoribbons. Appl. Phys. Lett. 2014, 105, 023101.

24. Yang, F.; Wang, X.; Zhang, D.; Yang, J.; LuoDa; Xu, Z.; Wei, J.; Wang, J.-Q.; Xu, Z.; Peng, F., et al. Chirality-Specific Growth of Single-Walled Carbon Nanotubes on Solid Alloy Catalysts. Nature 2014, 510, 522-524.

25. Omachi, H.; Nakayama, T.; Takahashi, E.; Segawa, Y.; Itami, K. Initiation of Carbon Nanotube Growth by Well-Defined Carbon Nanorings. Nature Chem. 2013, 5, 572-576.

26. Abbas, A. N.; Liu, G.; Narita, A.; Orosco, M.; Feng, X.; Müllen, K.; Zhou, C.

Deposition, Characterization, and Thin-Film-Based Chemical Sensing of Ultra-Long Chemically Synthesized Graphene Nanoribbons. J. Am. Chem. Soc. 2014, 136, 7555-7558.

27. Qian, G.; Wang, Z. Y. Near-Infrared Organic Compounds and Emerging Applications. Chem. Asian J. 2010, 5, 1006-1029.

28. Jorio, A.; Saito, R.; Dresselhaus, G.; Dresselhaus, M. S. Raman Spectroscopy in Graphene Related Systems. Wiley-VCH Verlag: Weinheim, 2011.

29. Liu, P.; Chen, Y.; Deng, J.; Tu, Y. An Efficient Method for the Preparation of Benzylic Bromides. Synthesis 2001, 14, 2078-2080.

30. Shifrina, Z. B.; Averina, M. S.; Rusanov, A. L.; Wagner, M.; Müllen, K. Branched Polyphenylenes by Repetitive Diels-Alder Cycloaddition. Macromolecules 2000, 33, 3525-3529.

31. Kumar, U.; Neenan, T. X. Diels-Alder Polymerization between Bis(cyclopentadienones) and Acetylenes. A Versatile Route to New Highly Aromatic Polymers. Macromolecules 1995, 28, 124-130.

32. Centrone, A.; Brambilla, L.; Renouard, T.; Gherghel, L.; Mathis, C.; Müllen, K.; Zerbi, G. Structure of New Carbonaceous Materials: The Role of Vibrational Spectroscopy. Carbon 2005, 43, 1593-1609.

33. Schmidt-Rohr, K.; Spiess, H. W. Multidimensional Solid-State NMR and Polymers. Academic Press: New York, 1994.

34. Shu, J.; Dudenko, D.; Esmaeili, M.; Park, J. H.; Puniredd, S. R.; Chang, J. Y.; Breiby, D. W.; Pisula, W.; Hansen, M. R. Coexistence of Helical Morphologies in Columnar Stacks of StarShaped Discotic Hydrazones. J. Am. Chem. Soc. 2013, 135, 11075-11086.

35. Brown, S. P.; Schnell, I.; Brand, J. D.; Müllen, K.; Spiess, H. W. An Investigation of $\pi-$ $\pi$ Packing in a Columnar Hexabenzocoronene by Fast Magic-Angle Spinning and DoubleQuantum 1H Solid-State NMR Spectroscopy. J. Am. Chem. Soc. 1999, 121, 6712-6718. 
36. Ochsenfeld, C.; Brown, S. P.; Schnell, I.; Gauss, J.; Spiess, H. W. Structure Assignment in the Solid State by the Coupling of Quantum Chemical Calculations with NMR Experiments: A Columnar Hexabenzocoronene Derivative. J. Am. Chem. Soc. 2001, 123, 2597-2606.

37. Hansen, M. R.; Feng, X.; Macho, V.; Müllen, K.; Spiess, H. W.; Floudas, G. Fast and Slow Dynamics in a Discotic Liquid Crystal with Regions of Columnar Order and Disorder. Phys. Rev. Lett. 2011, 107, 257801.

38. Feike, M.; Demco, D. E.; Graf, R.; Gottwald, J.; Hafner, S.; Spiess, H. W. Broadband Multiple-Quantum NMR Spectroscopy. J. Magn. Reson. Ser. A 1996, 122, 214-221.

39. Saalwächter, K.; Lange, F.; Matyjaszewski, K.; Huang, C.-F.; Graf, R. BaBa-xy16: Robust and Broadband Homonuclear DQ Recoupling for Applications in Rigid and Soft Solids up to the Highest MAS Frequencies. J. Magn. Reson. 2011, 212, 204-215.

40. Saalwächter, K.; Schnell, I. REDOR-Based Heteronuclear Dipolar Correlation Experiments in Multi-Spin Systems: Rotor-Encoding, Directing, and Multiple Distance and Angle Determination. Solid State Nucl. Magn. Reson. 2002, 22, 154-187.

41. Giordani, S.; Bergin, S. D.; Nicolosi, V.; Lebedkin, S.; Kappes, M. M.; Blau, W. J.; Coleman, J. N. Debundling of Single-Walled Nanotubes by Dilution: Observation of Large Populations of Individual Nanotubes in Amide Solvent Dispersions. J. Phys. Chem. B 2006, 110, 15708-15718.

42. Hernandez, Y.; Nicolosi, V.; Lotya, M.; Blighe, F. M.; Sun, Z.; De, S.; McGovern, I. T.; Holland, B.; Byrne, M.; Gun'Ko, Y. K., et al. High-Yield Production of Graphene by LiquidPhase Exfoliation of Graphite. Nature Nanotech. 2008, 3, 563-568.

43. Saito, R.; Hofmann, M.; Dresselhaus, G.; Jorio, A.; Dresselhaus, M. S. Raman Spectroscopy of Graphene and Carbon Nanotubes. Adv. Phys. 2011, 60, 413-550.

44. Detailed Raman studies on GNR 1 will be published elsewhere.

45. Ulbricht, R.; Hendry, E.; Shan, J.; Heinz, T.; Bonn, M. Carrier Dynamics in

Semiconductors Studied with Time-Resolved Terahertz Spectroscopy. Rev. Mod. Phys. 2011, 83, 543-586.

46. Jensen, S. A.; Ulbricht, R.; Narita, A.; Feng, X.; Müllen, K.; Hertel, T.; Turchinovich, D.; Bonn, M. Ultrafast Photoconductivity of Graphene Nanoribbons and Carbon Nanotubes. Nano Lett. 2013, 13, 5925-5930.

47. Tielrooij, K. J.; Song, J. C. W.; Jensen, S. A.; Centeno, A.; Pesquera, A.; Zurutuza Elorza, A.; Bonn, M.; Levitov, L. S.; Koppens, F. H. L. Photoexcitation Cascade and Multiple Hot-Carrier Generation in Graphene. Nature Phys. 2013, 9, 248-252.

48. Ueda, A.; Matsuda, K.; Tayagaki, T.; Kanemitsu, Y. Carrier Multiplication in Carbon Nanotubes Studied by Femtosecond Pump-Probe Spectroscopy. Appl. Phys. Lett. 2008, 92, 233105.

49. Pijpers, J. J. H.; Ulbricht, R.; Tielrooij, K. J.; Osherov, A.; Golan, Y.; Delerue, C.; Allan, G.; Bonn, M. Assessment of Carrier-Multiplication Efficiency in Bulk PbSe and PbS. Nature Phys. 2009, 5, 811-814.

50. Thakur, R. S.; Kurur, N. D.; Madhu, P. K. Swept-Frequency Two-Pulse Phase Modulation for Heteronuclear Dipolar Decoupling in Solid-State NMR. Chem. Phys. Lett. 2006, 426, 459-463.

51. Paul, S.; Kurur, N. D.; Madhu, P. K. On the Choice of Heteronuclear Dipolar Decoupling Scheme in Solid-State NMR. J. Magn. Reson. 2010, 207, 140-148.

52. Frye, J. S.; Maciel, G. E. Setting the Magic Angle Using a Quadrupolar Nuclide. J. Magn. Reson. 1982, 48, 125-131. 
53. Hayashi, S.; Hayamizu, K. Chemical Shift Standards in High-Resolution Solid-State NMR (1) ${ }^{13} \mathrm{C},{ }^{29} \mathrm{Si}$, and ${ }^{1} \mathrm{H}$ Nuclei. Bull. Chem. Soc. Jpn. 1991, 64, 685-687.

54. Morcombe, C. R.; Zilm, K. W. Chemical Shift Referencing in MAS Solid State NMR. J. Magn. Reson. 2003, 162, 479-486.

\section{TOC GRAPHIC}

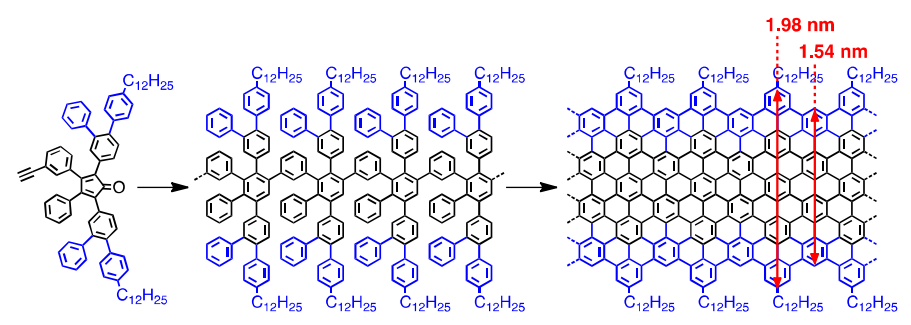

